\title{
Casa e vicinato a Bombay: la città degli immigranti
}

\section{Home and Neighbourhood in Bombay: the Immigrants' City}

Rossella Ciocca [rciocca@unior.it]

Università di Napoli “L’Orientale”, Italia

\begin{abstract}
RiAssunto
Mumbay è una megalopoli globale, capitale economica e culturale dell'India neo-liberista, è centro di flussi migratori mastodontici che ne hanno negli ultimi decenni stravolto i lineamenti. Questo saggio si propone un'analisi della sua identità problematica e contraddittoria attraverso esempi tratti dalla cosiddetta Bombay fiction: una letteratura non solo ambientata in città ma che ha per oggetto la città stessa. Cercando di coglierne il profilo multiculturale, le dimensioni della casa e del vicinato vengono prescelte per investigare le connessioni inter e intra comunitarie a ridosso di tre romanzi anglofoni: Ravan and Eddie (1995) di Kiran Nagarkar; Sacred Games (2006) di Vikram Chandra; Last Man in Tower (2011) di Aravind Adiga.
\end{abstract}

\section{Parole Chiave}

Narrativa di Bombay; Paesaggio migrante; Città globale; Casa; Vicinato

\begin{abstract}
Mumbai is a new kind of world place, not only the capital of modernizing India but the very embodiment of its contradictory, neo-liberal advance; a transnational and trans-local contact zone which condenses all the contradictions and the cultural varieties of contemporary global megalopolises. This paper seeks to articulate a description of the city viewed through the lens of its migrant flows, on the one hand, and its narrative renditions, on the other. With the intention to draw a map of its multicultural, problematic identity, the analysis will concentrate upon home and neighbourhood seen as key topics to investigate the connections within and between the different communities. Examples will be drawn from the field of the Anglophone contemporary Bombay novel, namely: Ravan and Eddie (1995) by K. Nagarkar; Sacred Games (2006) by V. Chandra; Last Man in Tower (2011) by A. Adiga.
\end{abstract}

\section{KEYWORDS}

Bombay fiction; Migrant landscape; Global city; Home; Neighbourhood

RICEVUTO 2015-08-31; ACCETTATO 2015-12-05 


\section{Città e paesaggio migrante}

La mappa metropolitana del presente, dove il futuro pare mostrare il suo volto più inquietante, è punteggiata da centri urbani cresciuti a dismisura, soprattutto in quella parte di mondo conosciuto come il Sud globalizzato. Ogni singola megacity è anche sempre più percepita come un nodo in una rete di connessioni attraverso cui, lungo una serie complessa di traiettorie sovrapposte, il locale finisce per partecipare al e costruire il globale. Una delle più significative linee di connessione tra le diverse realtà urbane è sicuramente rappresentata dalle comunità diasporiche e migranti, sia a livello transnazionale che translocale, quando, come ad esempio nella regione meridionale dell'Asia, la maggior parte dei flussi oltre confine avviene a livello macro-regionale e intra-nazionale. Se uno degli effetti dello sviluppo di tali reticolati, a ridosso dei fenomeni di spostamento umano di massa, è stato quello di depotenziare e confondere a livello globale la demarcazione geografico-economica tra Nord sviluppato e Sud arretrato, a livello locale, la città diviene a sua volta uno spazio reticolato di connessione. In tal senso essa si costituisce quale punto focale del paesaggio migrante e sito privilegiato per incontrare la differenza, l'alterità, e, attraverso tutto il graduale spettro di possibilità intermedie, esperire forme di ibridazione culturale.

La megalopoli è oggi, come mai prima, una cosmopoli, una polis con dentro l'intero cosmo: etnico, culturale, religioso, linguistico, ma anche inusitata espressione di collassamento spazio-temporale. Essa si costituisce come zona di contatto (Pratt 1992) per eccellenza, una dimensione congiunturale che mette in crisi tutta una serie di dicotomie geografiche e storiche tradizionali per creare quelle che Varma definisce le "geografie simultanee" (2012:2) di un Nord e un Sud intercettati continuamente da un passato e un presente parimenti coesistenti. Basti pensare alle baraccopoli, veri e propri villaggi edificati fin nel centro delle megalopoli, in cui le nozioni di centro e di periferia non tengono più, o anche alla contemporaneità metropolitana tra segmenti temporali generalmente non consecutivi con forme di produzione e sussistenza di tipo pre-capitalistico rinvenibili accanto all'ipermodernità tecnologica delle cittadelle finanziarie (Sassen 2005) ${ }^{1}$.

Il paesaggio diasporico e migratorio è dunque significativamente paesaggio urbano, e tale paesaggio risulta esponenzialmente moltiplicato e differenziato attraverso il sistema plurale delle affiliazioni trans-locali dei gruppi migranti. Per sistema delle affiliazioni s'intende non solo il primario codice identificativo, razziale, etnico, religioso e di genere, ma anche tutta una più fitta trama di aderenze di natura affettiva e immaginativa. In realtà il senso di appartenenza o dis-appartenenza, prima che attraverso il sistema delle macro-categorie di individuazione collettiva, passa per il più sottile ma fittamente intrecciato ordito di eperienze materiali o sensoriali legate alle pratiche del vivere quotidiano. Come testimoniano, in effetti, gli studi più

1 Nella compresenza di settori leader del capitalismo globalizzato e di una sempre più massiccia presenza di porzioni di popolazione svantaggiata rappresentata in primis da immigranti, rifugiati, clandestini, Sassen ha per prima visto la città come sito strategico di una nuova lunga serie di conflitti e contraddizioni: "If we consider that global cities concentrate both the leading sectors of global capital and a growing share of disadvantaged populations (immigrants, many of the disadvantaged women, people of color generally, and, in the megacities of developing countries, masses of shanty dwellers) then we can see that cities have become a strategic terrain for a whole series of conflicts and contradictions"(2005: 39). 
recenti sui fenomeni diasporici ${ }^{2}$, che hanno ampliato il loro raggio d'indagine alle dimensioni materiali delle pratiche abitative e delle architetture domestiche o a quelle sensoriali legate alle culture della cucina e del cibo in generale, le identità migranti non risultano articolate esclusivamente attorno al loro status, generalmente di non cittadinanza, ma molto più specificate a ridosso di un dominio di tipo affettivo e sensoriale, una sorta di fluido aggregato fatto di immaginazioni, abitudini ed affetti.

Strumento di registrazione privilegiato di una tale dimensione è sicuramente il piano narrativo che sulla trama del vissuto intesse le proprie storie. Le città come sito di narrazione è un topos caro agli studi letterari e culturali ma oggi, soprattutto nella sua articolazione transnazionale e trans-locale, come momento di ricerca di una possibile sintesi tra spinte identificative diverse e spesso in tensione tra di loro, esso tende a diventare sintomatico anche in relazione a campi disciplinari molto più ampi e differenziati e ad acquisire un peso che sicuramente travalica l'estetico e si intreccia fortemente con il politico.

Nella letteratura della città, come locus che ricolloca il migrante a partire dalla sua dislocazione e lo ri-territorializza a partire dal suo spaesamento e dal suo oscillare tra mobilità e stanzialità, l'immaginario narrativo è spesso dunque fortemente collegato alle, generalmente limitate, possibilità di insediamento e rilocalizzazione, enfatizzando o comunque comprendendo centralmente riflessioni e spunti narrativi relativi alle dimensioni della casa e del vicinato. L'idea portante della trans-località è in effetti abilmente sintetizzata nella definizione di Brickell e Datta di "ri-localizzazione nella mobilità" . Le narrazioni interessate a sviluppare la prospettiva transnazionale/translocale del soggetto migrante si articolano dunque all'interno di un paesaggio in cui il senso del movimento e della discontinuità, della separazione e della perdita si stemperano materialmente e affettivamente in uno scenario fatto di luoghi e posti, magari temporanei, dove comunque si cerca di ri-sperimentare il senso della casa, anche se all'interno della dinamica, questa volta inversa, dal globale al locale.

In questo saggio ci si concentrerà su una particolare megalopoli asiatica, Mumbai, sulla narrativizzazione del suo carattere eminentemente immigratorio rappresentata dalla cosiddetta Bombay fiction ${ }^{4}$ e su alcuni luoghi fisici in cui le soggettività trans-locali si sono confrontate con l'ambivalenza mobilità/rilocalizzazione e la costruzione di un'affettività domestica legata all'idea di 'casa' e 'vicinato'.

2 Inizialmente gli studi che si sono occupati di fenomeni transnazionali hanno posto l'accento sulla messa in discussione di categorie. una volta ritenute stabili, quali stato-nazione o confine nazionale, occupandosi fondamentalmente di processi di de-territorializzazione. Gradualmente, mentre veniva decostruito il concetto di 'radicamento' come costruito esclusivamente sul nesso trà identità e territorio è emersa la tendenza a riconcettualizzare il contesto locale come fondamento cogente anche rispetto a fenomeni di transnazionalismo. Gli studiosi di fenomeni migratori e diasporici hanno, negli ultimi anni, rivolto una nuova attenzione a forme di articolazione identitaria in relazione a pratiche di ri-territorializzazione all'interno di spazi come la città, il quartiere e la casa dando vita alla concettualizzazione di una sorta di trans-nazionalismo territorializzato, concentrato sul nesso tra mobilità e residenzialità. Vedi ad esempio Brickell, Datta (2011); Freitag, von Oppen (2010); Cloke, Johnston (2005).

3 Brickell e Datta usano l'espressione "situated-ness during mobility" (2011: 3). Tutte le traduzioni dei testi in inglese sono mie. Le pagine delle citazioni fanno pertanto riferimento all'edizione inglese indicata in bibliografia.

4 La categoria della Bombay/Mumbai Fiction, ormai correntemente usata dalla critica, è stata coniata negli ultimi anni in riferimento al fenomeno di esplosione narrativa che ha visto la città protagonista non solo in quanto sfondo delle vicende raccontate ma anche come ispiratrice di storie. Vedi in particolare Prakash, ma anche Gopal (2009), Ciocca (2008), Dawson Varughese (2013). 


\section{Mumbai, città migrante}

Mumbai è una città postcoloniale e una città globale: una megalopoli dall'inconfondibile skyline e la più massiccia estensione di baraccopoli dell'Asia e forse del mondo. I suoi slums contano almeno nove milioni di abitanti ma molti suoi "residenti" non hanno neanche un tetto di lamiera sulla testa e si arrangiano sotto i cavalcavia o semplicemente sui marciapiedi. La città ospita fianco a fianco una grande varietà di gruppi etnici, diverse culture, religioni, classi e caste. Dal 1947 in poi è meta continua di flussi migratori dalle campagne, dai villaggi, dalle regioni limitrofe e da tutti gli stati indiani, dalle altre città del subcontinente e da tutta l'area dell'Asia meridionale. Il suo monumento simbolo è the Gateway of India, l'imponente arco di basalto prospicente il porto costruito nel 1927, per commemorare la visita di Giorgio V, mentre venti anni dopo, avrebbe rappresentato la porta di uscita delle truppe britanniche che lasciavano l'impero. Oggi esso marca la città come snodo di passaggio: sia meta di arrivo che città di partenza, essendo Mumbai, oltre a una destinazione immigratoria, anche uno dei principali varchi dell'emigrazione sud asiatica, prima verso l'Africa orientale, ora soprattutto verso i paesi del Golfo, il Pacifico, le Americhe e anche l'Europa.

Perché questa confluenza verso la città? Subito dopo l'indipendenza, Bombay aveva rappresentato una delle incarnazioni del progetto nazionalista di un'India che sapesse guardare al mondo fuori di sé e che si incamminasse lungo un percorso di protagonismo sul fronte della modernità senza dimenticare il mandato del progetto universalista dell'inclusione sociale e dell'emancipazione dalle strettoie confessionali e castali. Bombay è la città che ha alimentato il sogno emancipativo dell'individuo che, scappando dalle campagne, cercava nell'anonimato della metropoli e nella fabbrica dei sogni di celluloide di Bollywood, la possibilità di una realizzazione anche individuale in una terra di pressioni comunitarie pesantissime. In una versione di cosmopolitismo universalistico, indebitato con la tradizione illuministica da un lato e col progetto anticoloniale, nazionalista, e statalista di Nehru dall'altro, Bombay aveva rappresentato la possibilità di costruzione nel nuovo stato-nazione di quella "unità nella diversità" di segno secolarizzato all'interno, non allineato e terzomondista in campo internazionale. In definitiva, pur con tutte le sue contraddizioni, Bombay, celebrata da Salman Rushdie come "la figlia bastarda di un matrimonio portoghese-inglese" (1996: 350) no, almeno fino agli anni Ottanta, un laboratorio metropolitano aperto e tollerante, ibridato e vivace, una zona di contatto multietnica, multiculturale e multiconfessionale.

A partire da metà anni Ottanta e mentre la città cominciava a diventare quel "ricettacolo rigonfio", così ben descritta da Sunil Khilnani (2003), in perenne stato di imminente cedimento, la svolta regionalista della destra Indù nella sua articolazione politica locale del partito fondamentalista dello Shiv Sena produceva un drastico cambiamento di clima facendo calare sulla aperta Bombay la cappa della mistica violenta e reazionaria dell'affiliazione di sangue-suolocredo religioso. L'infrangersi del sogno cosmopolita con la nascita di un nuovo parrocchialismo, aggressivo e razzista, ha poi portato la città, nel frattempo ribattezzata Mumbai ${ }^{6}$, a diventare

5 "The bastard child of a Portuguese-English wedding" (350).

6 Conosciuta per quattro secoli col nome di Bombay (dizione anglicizzata delloriginale portoghese Bombaim presumibilmente da Bom Bahia), la città, seguendo una generale politica di 're-induizzazione' del paese, viene rinominata Mumbai in onore della dea Indù Mumbadevi nel 1995 da una coalizione guidata dall'estrema destra localista dello Shiv 
teatro di una serie di sommosse e di attentati su base confessionale, ogni volta con centinaia di vittime. Dalla prima deflagrante esplosione di violenza nel 1992-93 e poi ripetutamente, nel 2006, fino all'attacco terroristico del 2008 e ancora del 2011, lungo il fronte dello scontro tra associazioni mafiose islamiche, servizi segreti stranieri e bande paramilitari induiste, l'attacco alla città e ai suoi civili si è concentrato su una serie di simboli urbanistici ${ }^{7}$, significanti di una Bombay globale, contaminata, multiconfessionale e turistica.

\section{Bombay Fiction}

Mumbai è una città altamente iconica: il suo immaginario urbano, con la silhouette di grattacieli su un'infinita distesa di tetti di lamiera, la vitalità della sua vita notturna, il lungomare che incornicia i quartieri alti ma ospita gratis gli abitanti degli slums, la movida hippy, la guerra tra gansters, è stato costruito nel tempo soprattutto attraverso Bollywood (Mishra 2002). Contemporaneamente, una più complessiva proliferazione narrativa, fatta di canzoni, serie televisive, giornalismo popolare, fumetti, graphic novels, short stories, e soprattutto romanzi, ha generato e alimentato nel tempo una corrente ininterrotta d'immagini e racconti riuniti sotto l'etichetta di Bombay fiction. Un flusso narrativo cui è spettato negli anni di tematizzare, elaborare criticamente e negoziare transizioni, sviluppi e soprattutto una serie di passaggi critici e turbolenti. Tutti o quasi connessi con l'esponenziale aumento demografico legato alla continua affluenza migratoria in città, accompagnando ed affettivamente marcando le varie cesure degli ultimi decenni: da città industriale post-indipendenza, ancora in qualche modo cosmopolita e accogliente, a metropoli sconquassata dalla violenza comunitaria degli anni ' 80 e ' 90 , fino alla megalopoli stravolta dalla febbre imprenditoriale neo-liberista del terzo millennio.

Tramite tre esempi narrativi, tra i mille possibili, si tenterà di ricostruire il clima legato alle vicende migratorie e ai processi di ri-territorializzazione nella mobilità, talvolta nella vera e propria precarietà stanziale, attraverso alcune forme abitative tipiche della città, e rappresentative del concetto di casa e di vicinato per gli immigrati. Il chawl o caseggiato popolare, il jhopadpatty o baraccopoli, e il tower block nella doppia accezione di condominio residenziale post-bellico e grattacielo super-lusso del terzo millennio. Il primo romanzo in questione è Ravan and Eddie, pubblicato da Kiran Nagarkar ${ }^{8}$ a metà anni '90 ma ambientato nella Bombay degli anni ‘60. La

Sena decisa a cancellare quante più tracce possibili del passato coloniale ma anche della vecchia idea di città aperta sia all'Occidente che ai massicci flussi migratori interni. Da allora nell'uso soprattutto degli intellettuali e degli artisti il nome di Bombay viene rivendicato come espressione di una dimensione più aperta e liberale della metropoli, in contrasto con le tendenze più scioviniste e intolleranti sviluppatesi negli ultimi decenni. Qui si useranno entrambe le dizioni per sottolineare proprio lo svilupparsi di una specie di doppia anima della città e la compresenza di fattori ambivalenti e contraddittori.

7 Tra questi si ricordano ad esempio la sede della Borsa (lo Stock Exchange), uno dei mercati più popolari e cosmopoliti (lo Zaveri Bazaar), l'aereoporto internazionale, il centro ebraico, un ospedale per donne e bambini, un collegio cattolico, una sala cinematografica, due tra i più famosi e rinomati alberghi internazionali (l'Oberoi e il Taj Mahal Hotel), il famoso Leopold Café, luogo d'incontro frequentato da viaggiatori e turisti di tutto il mondo.

8 Kiran Nagarkar è tra i più interessanti scrittori indiani che si muovono nella dimensione del bilinguismo. Considerato uno dei più innovatori autori in lingua Marathi, Nagarkar ha pubblicato anche in inglese, sia traducendo i suoi stessi lavori dal marathi sia producendo direttamente in questa lingua. Nel caso di questo romanzo, il progetto iniziale riguardava la sceneggiatura di un cosiddetto Hindi film, trasformatosi nel corso degli anni in un lungometraggio in 
storia di due bambini, Ravan, di religione induista, e il cattolico Eddie, gemellati da un destino parallelo, si svolge in una specifica istituzione residenziale di Bombay: il chawl, espressione abitativa tipica della fase post-indipendenza quando la città si sviluppa come centro di produzione dell'industria tessile attirando maestranze soprattutto dagli stati limitrofi. È questa la fase in cui si mettono in campo vari timidi tentativi di piani regolatori e una serie di ambiziosi, quanto inconcludenti, progetti di sviluppo residenziale in chiave organica e complessiva così ben ricostruiti da Gyan Prakash (2010, cap. 7) nella sua "storia di una città incantata". A livello di edilizia popolare, il chawl si caratterizza come un lungo caseggiato, spesso a forma di croce e a più piani, con un corridoio centrale ai lati del quale si aprono delle stanze corrispondenti ai singoli appartamenti, composti di un unico ambiente senza servizi.

Ogni stanza misurava 12 piedi di larghezza e 24 di profondità con una partizione di legno che separava il soggiorno-letto-studio-biblioteca-area giochi, o quel che sia, dalla cucina che diventava pranzo che diventava letto che diventava salotto, che diventava spogliatoio, che diventava bagno ... (Nagarkar 1996: 67) ${ }^{9}$

L’essenza della vita nel chawl, dice l'autore, è che ogni cosa è di 'pubblico dominio':

Quando ti trovi costantemente testa a testa con gli altri, è quasi impossibile riuscire a spidocchiarti senza prendere nel frattempo qualche piccolo ospite dai tuoi vicini. $(54)^{10}$

Eppure la prossimità spaziale non significa necessariamente intimità o condivisione. L'apparente promiscuità risulta in realtà attraversata da una serie di linee, invisibili ma insormontabili, di delimitazione ad organizzare la coabitazione secondo una geografia complessamente e talvolta anche contraddittoriamente gerarchica. Una prima demarcazione separa i Bombayites di lungo corso dagli immigrati più recenti. La divisione successiva riguarda la religione, in questo caso la maggioranza è Hindu e la minoranza è cattolica, proveniente dallo stato di Goa che perde l'indipendenza nel 1961. Le vite delle due comunità procedono parallelamente e possono anche, come accade ai due protagonisti, non attraversarsi mai.

Gli hindu e i cattolici nei caseggiati del Dipartimento Centrale del Lavoro di Bombay (e forse di qualsiasi altra parte in India) avrebbero potuto vivere anche su pianeti diversi. Si vedevano ogni giorno, occasionalmente si scambiavano un saluto, ma le loro vite si incrociavano molto raramente. $(15)^{11}$

inglese, poi in un romanzo incompleto in marathi ed infine in un romanzo di stampo modernista scritto in un eclettico indian-english.

9 "Each room was twelve feet wide and twenty-four feet deep with a wooden partition separating the drawingroom-cum-bedroom-cum study, library, playpen or whatever from the kitchen which doubled as dining-room, bedroom, dressing-room, and bathroom..."

10 "When you are constantly in each other's hair, it's almost impossible to get the lice out of your own hair without picking a few out of somebody else's."

11 "The Hindus and Catholics in Bombay's CWD chawls (and perhaps almost anywhere in India) may as well have lived on different planets. They saw each other daily and greeted each other occasionally, but their paths rarely crossed." 
Altre traiettorie divisive sono articolate su base castale. L'accesso alla preziosissima e scarsissima risorsa dell'acqua mette in chiaro i codici non scritti di chi vanta diritti e chi no. "Quella gente", come vengono chiamati i componenti delle cira 300 famiglie di intoccabili che occupano il pian terreno, e le cui sole ombre sono considerate contaminanti, avevano accesso a un paio di rubinetti condominiali (i cattolici ne avevano 6), ma:

Quando l'acqua era poca o i giornali ne annunciavano la mancanza per il giorno successivo, gli intoccabili venivano scacciati dai loro rubinetti e l'acqua veniva raccolta da questi dopo che la prima donna della fila ne avesse, con un gesto automatico, gettato alcune gocce in direzione della bocchetta d'ottone del rubinetto, per renderla pulita ed utilizzabile. (p. 71) ${ }^{12}$

Ovviamente la vita nel chawl rispetta la differenza tra maschi e femmine, e persino quella tra chi ha la pelle più chiara - "Se si era di carnagione chiara, era ovvio essere considerati belli" - e chi ha la sfortuna di nascere scuro - "Essere nati di carnagione scura significava essersi macchiati del peccato originale"13 - ma soprattutto un altro grande fronte di divisione è rappresentato dal linguaggio. Gli induisti parlano marathi, i cattolici il konkani a casa, ma fuori comunicano in inglese, la lingua che fa la differenza:

L'inglese era la spina nel fianco degli induisti. La sua assenza la loro croce, la loro umiliazione e la fonte del loro perenne senso di inferiorità e inadeguatezza. [...] L'inglese era il grande livellatore, la medicina degli induisti cui faceva provare cosa significava diventare intoccabili. (179) ${ }^{14}$

Il romanzo si sviluppa in guisa di una serie di riflessioni sul 'vicinato' in una condizione abitativa che in realtà è solo una versione in piccolo dell'intera città, e dell'intera nazione, ognuna a vari livelli stretta nella contraddizione tra l'aspirazione a realizzare forme avanzate di pluralismo secolarizzato e l'incapacità di superare avativistiche forme di diffidenza tra le comunità. Nagarkar riflette in maniera sardonica, spesso grottesca, sui temi dell'identità e delle relazioni inter-gruppali in una città che è al tempo stesso definita e disturbata dalla costitutiva pluralità dei suoi mondi (Gopal 2009: 137) che però, pur non mischiandosi, appaiono ancora capaci di vivere fianco a fianco abbastanza pacificamente.

Nel giro di un paio di decadi, il volto tutto sommato benigno e progressivo di Bombay cambierà traumaticamente, la città vedrà la crisi del suo apparato industriale e, contraddittoriamente, mentre chiudono le fabbriche esplode il fenomeno dell'urbanizzazione selvaggia con ondate migratorie, spesso clandestine, sempre più ampie da tutto il subcontinente e dalle nazioni confinanti. Le ondate di nuovi poveri daranno vita ad immense baraccopoli, come ad

12 "When the water was short or if the papers gave notice that there was going to be a water-cut the next day, the untouchables were shooed away from their taps and water filled from them after the first woman in the queue perfunctorily threw a few drops in the direction of the brass nozzle of the faucet to make it clean and usable."

13 "If you were fair, it was obvious you were beautiful"; “To be dark was to have committed original sin" (p. 84).

14 "English was the thorn in the side of the Hindus. Its absence was their cross, their humiliation and the source of their life-long inferiority and inadequacy. [...] It was the great leveller. It gave caste-Hindus a taste of their own medicine. It made them feel like untouchables." 
esempio Dharavi, tristemente famosa come il più grande slum del mondo ${ }^{15}$, cancellando dalla città qualsiasi residua velleità di sviluppo organico e controllato.

Sacred Games, di Vikram Chandra (2006), una sorta de Il Padrino in salsa Masala, ben rappresenta il clima esasperato ed esagitato degli anni Ottanta e Novanta, mostrando una città sconvolta da continue eruzioni di violenza; violenza settaria e fondamentalista; violenza criminale delle bande, spesso organizzate su base confessionale, che si combattono per la supremazia nei traffici illeciti, toccando in modo anche profondo i mondi della politica e dello spettacolo; la violenza della polizia che per mantenere un minimo di controllo finisce per ricorrere alle stesse tattiche da guerriglia delle varie associazioni a delinquere.

I destini violenti di tutti questi attori, come nella Chicago degli anni '20 risulteranno in più di un'occasione intrecciati con la pulsante vita dell'underworld clandestico degli slums. Un universo di povertà e speranze destinate quasi sempre a naufragare nell'indigenza, nella brutalità, spesso nella morte.

[...] c'era un furioso senso di disprezzo per gli abitanti delle baraccopoli che lasciavano lo sporco e l'immondizia accumularsi appena fuori le loro case, che lasciavano le loro figlie piccole fare i bisogni dove giocavano i bambini maschi. Questa è la gente che rovina Mumbai [...] tutta questa gente che arriva dal Bihar, dall'Andhra Pradesh o dal fottuto Bangladesh e vive qui come le bestie. Questi in particolare erano certamente del fottuto Bangladesh [...] anche se senza dubbio avevano documenti che dicevano che venivano dal Bengala, che ognuno di loro era un cittadino indiano certificato! In ogni caso, non c'era alcun posto nel loro acquoso delta dove rimandarli indietro [...]. Arrivavano qui a migliaia per lavorare come servi o per le strade o nei cantieri. E ora qui ce n'era uno morto. (Chandra 2007: 20) ${ }^{16}$

Molti giovani immigrati clandestini, sedotti da stili di vita irragiungibili se non attraverso le scorciatoie del crimine e della malavita organizzata, finiscono infatti per incorrere in morti violente e premature. Quello trovato nella baracca con la gola tagliata e un dito mancante, "[...] non serviva cercarlo, in giro c'erano dei topi" (21), probabilmente aveva cominciato a fare rapine per procurare una dote alle sue sorelle, ma poi,

Il ragazzo morto aveva voluto di più del matrimonio per le sorelle, aveva voluto un televisore e una cucina a gas e una pentola a pressione e una casa più grande. Senz’ombra di dubbio aveva sognato una automobile nuova di zecca ... quel che aveva sognato non era impossibile, c'erano uomini come Ganesh Gaitonde e Suleiman Isa, che avevano cominciato con piccoli

15 Presso il grande pubblico, Dharavi diventerà famosa per aver ospitato le riprese delle scene nella baraccopoli nel film del regista Danny Boyle Slumdog Millionaire (2008), vincitore di 8 premi Oscar.

16 " $[. .$.$] there was furious contempt for jhopadpatti-dwellers who let dirt and filth and garbage pile up not two feet$ from their own doors, who let their little daughters squat to make a mess where their sons played. These are the people who ruin Mumbai, [...] these ganwars who come from Bihar or Andhra or maderchod Bangladesh and live like animals here. These were indeed from maderchod Bangladesh [...] although they all no doubt had papers that said they were from Bengal, that each was a bona fide Indian citizen. Anyway, there was nowhere in their watery delta to send them back to $[\ldots]$. They came in their thousands, to work as servants and on the roads and on the construction sites. And one of them was dead here." 
furtarelli e avevano continuato fino a possedere flotte di Opel Vectras e di Honda Accords. $(226)^{17}$

In effetti, la storia di Sacred Games è quella di un gangster che viene dai bassifondi e sui bassifondi costruisce la sua iniziale fortuna, dopo aver investito la sua prima somma di denaro, fortunosamente acquisita, nella costruzione di siti illegali destinati agli immigrati, spesso clandestini, della città. Prima ancora che fossero completate c'era già la fila per ottenere le casupole appene erette in modo illegittimo sui suoli totalmente privi di permessi.

Su e giù per la strada, la baraccopoli cresceva, si arrampicava su per la collina, ogni giorno sembrava allargarsi. Sin dall'inizio avevamo Dalit e altri appartenenti alle caste basse, i Marati e i Tamil, i Bramini e i Mussulmani. (111) ${ }^{18}$

Interessante in questo resoconto è notare come le categorie di casta (Dalit, Bramini) si mischino e sovrappongano a quelle geografiche (Marati, Tamil), e a quelle religiose (Mussulmani). In effetti, anche nell'apparente caos della baraccopoli, così come già nel chawl, un'invisibile geografia comunitaria si rivela operante lungo linee di appartenenza identitaria.

Le comunità tendevano a raggrupparsi, vicolo per vicolo. Alle persone piace stare con quelli che conoscono, il simile cerca il simile... in questa giungla dove un uomo può perdere persino il proprio nome e diventare qualcos'altro, i più umili degli umili si cercavano tra loro per vivere insieme in un orgoglioso comune squallore. (pp. 111-12) ${ }^{19}$

Nel romanzo di Chandra, come in tanti altri esempi della Bombay fiction ${ }^{20}$ e docufiction ${ }^{21}$, la vita nello slum viene descritta anche nella sua complessità relazionale e organizzativa, nelle sue capacità economiche, sia commerciali che, in alcuni settori, anche produttive, e soprattutto come centro funzionale alla dinamica politica della città, fungendo in tutte le elezioni come fondamentale serbatoio di voti e manovalanza, oltre che della criminalità, anche per le organizzazioni paramilitari dei vari partiti fondamentalisti.

In quegli anni, gli slums, da quelli di centinaia di migliaia di baracche a quelli che ne contano anche solo qualche decina, si diffondono dappertutto, nell'entroterra, vicino agli aeroporti, in periferia ma anche al centro, vicino al mare, sotto ogni cavalcavia, in ogni interstizio, fino

17 "The dead boy had wanted more than marriage for his sisters, he had wanted a television set and a gas range and a pressure cooker and a larger house. No doubt he had dreamed of a brand-new car [...]. What he had dreamed was not impossible, there were men like Ganesh Gaitonde and Suleiman Isa, who had begun with petty thefts and had gone on to own fleets of Opel Vectras and Honda Accords."

18 "Up and down the road the basti spread, and it went climbing up the hill, it seemed to grow every day. Right from the beginning, we had Dalits and OBCs, Marathas and Tamils, Brahmins and Muslims."

19 "The communities tended to cluster together, lane by lane. People like to stay with those they know, like seeks like [...] in this jungle where a man can lose his name and become something else, the lowest of the low will seek his own kind, and live with them in proud public squalor."

20 Sulla narrativizzazione degli slum vedi in particolare Ciocca (2008).

21 Per quest'ultima categoria si vedano in particolare Mehta (2005), e più recentemente Boo (2012). 
ad occupare i tetti dei grattacieli dove abitano i ricchi. ${ }^{22}$ In questa fase la crescita di Mumbai sembra inarrestabile e il suo modello un inurbamento parassitario, mostruoso e incoercibile destinato ad ingoiare la città coloniale e storica con il suo lungomare, la cosiddetta "collana di perle", e i quartieri eleganti. Lo slum diventa l'immagine simbolo della città.

Con tratti talvolta apocalittici e coll'andamento di una detective story, che è non solo romanzo di genere ma anche reportage su una città in tumultuosa espansione, il romanzo di Chandra ben racconta il passaggio dagli anni 60-70 a quella dell'esplosione demografica delle successive decadi. Come i vari sobborghi finiscano per saldarsi in un'unica infinita distesa di nuovi insediamenti abitativi, la stragrande maggioranza dei quali fatta di casupole fatiscenti di cartone e lamiera.

A sinistra c'era un lungo segmento grigio, l'edificio a fianco, trasformato dalla sagoma della finestra in un'astrazione di scanalature, a destra ciò che era considerato tenebra ma che pian piano sotto lo sguardo si disintegrava in un'amorfa quanto inarrestabile luminescenza giallognola. Sartaj sapeva da dove veniva e cosa l'aveva prodotta, ma come sempre ne fu annichilito. Si ricordava di quando poteva giocare per le strade di Dadar [...] e la sensazione di poter tener nel suo cuore tutta la città, da Colaba fino a Bandra. Ora era diventata troppo grande, gli si sottraeva, mentre famiglia dopo famiglia dopo famiglia si aggiungevano l'una all'altra fino alla creazione di quell'infinito e freddo bagliore, impossibile da conoscere o da evitare. $(23)^{23}$

Ma al volgere del terzo millennio, nel profilo urbanistico schizofrenico di città verticale che si erge sulla sconfinata estensione orizzontale delle baraccopoli, un'ulteriore fase sembra essersi aperta e quella che Arjun Appadurai (2000) definisce una vera e propria isteria abitativa prende piede, stalvolta in parte a scapito proprio degli slums e a favore di sempre più arditi piani di cosiddetta riqualificazione abitativa. All'argomento Aravind Adiga ${ }^{24}$ dedica il suo secondo romanzo intitolato Last Man in Tower (L'ultimo uomo sulla torre): la torre sta per un fatiscente condominio post-indipendenza, una volta signorile ma ormai circondato dagli slums, che deve fare posto a due nuovi grattacieli superlusso in un ambizioso progetto di speculazione edilizia che, dal centro della città, dove non c'è più spazio, è obbligata a spostarsi verso le periferie.

Bombay si ripiegava, come uno yogy, su se stessa, mentre il suo centro si spostava dal sud, dove non c'era più spazio dove espandersi, verso questa terra paludosa vicino all'aeroporto. Nuovi centri finanziari si andavano aprendo ogni mese [...] American Express, ICICI Bank, HSBC,

22 Essendo alle maestranze che lavoravano agli ultimi piani proibito l'uso degli ascensori, riservato ai materiali di costruzione, queste tendevano a rimanere su e a sviluppare, anche dopo la chiusura del cantiere, piccole comunità di residenti clandestini con tanto di tende da campo, piccole cucine e persino animali da cortile. Vedi a proposito i romanzi di Gregory David Robert (2004) e Altaf Tirewala (2007).

23 "To the left there was a long grey sliver, the building next door, turned by the window frame into a crenellated abstraction, and to the right what was called darkness, what disintegrated softly under the eye into an amorphous and relentless yellow illumination. Sartaj knew where it came from, what made it, but as always he was awed by it. He remembered playing cricket on a Dadar street [...] and the feeling that he could hold the whole city in his heart [...]. Now it was too vast, escaped from him, each family adding to the next and the next until there was that cool and endless glow, impossible to know, or escape."

24 Vincitore del prestigioso Booker Prize nel 2008 per il suo primo romanzo, The White Tiger. 
Citibank, se ne potrebbe menzionare una a caso, e la ricchezza delle loro volte, come burro su un piatto bollente, si scioglieva cadendo a rivoli sulle baraccopoli, arricchendo qualcuno, bruciando qualcun altro tra i suoi residenti. (Adiga 2012: 37) ${ }^{25}$

Nella città con una delle maggiori concentrazioni al mondo di abitanti per metro quadrato, e anche con i prezzi più alti, il tema dell'ansia residenziale scatena una guerra per lo spazio (Appadurai 2000: 635-44): da un lato i poveri, con le loro baracche, dall'altra il potere dei soldi e dell'interesse privato. Il vero fatto nuovo è costituito sicuramente dalla svolta neo-liberista, che ha di fatto convertito la pressione demografica a vantaggio di un agonismo edificativo che tende persino a superare le primitivistiche catene di solidarietà basate su connessioni etniche o religiose o di casta e a introdurre un più generalizzato clima di guerra di ognuno contro tutti.

[...] le cose erano cambiate. Si era ora in una stagione di 'potere della volontà' a Bombay: la combinazione di corruzione, filantropia ed inerzia che li aveva protetti per tanto tempo si stava disintegrando. Un nuovo funzionario aveva ricevuto l'incarico di sgomberare le baraccopoli illegali della città. Aveva spazzato via migliaia di baracche a Thane e ora prometteva di fare lo stesso a Mumbai. (Adiga 2012: 143)26

Il romanzo di Adiga coglie gli spasmi di questa nuova atmosfera che va riconfigurando anche in India la modernità come progressiva soggezione dell'idea di collettività alla logica del mercato e dei capitali.

[...] il governo sta cominciando la riqualificazione di Dharavi. La baraccopoli più grande dell'Asia diventerà la baraccopoli più ricca dell'Asia. Quest'area ribollirà di quattrini. (55) ${ }^{27}$

E mentre alcuni fortunati proprietari di baracche diventano milionari in cambio dei loro pezzettini di suolo edificabile ("Oggi dio è andato in visita negli slum", 36) ${ }^{28}$, altri vengono spazzati via dai bulldozer, le loro capanne spianate via dalla sera alla mattina (38).

Mumbai si apparenta alle altre megalopoli del pianeta, connesse in rete dalla new economy, seguendo il medesimo schema all'opera in ciacuna di esse:

[...] in cui baraccopoli e quartieri poveri vengono spazzati via per fare posto a uffici e centri commerciali, dove il terreno agricolo è fagocitato da una espansione urbana in perenne crescita e dove una nuova elite manageriale e tecnocratica si sovrappone alle vecchie dirigenze cercando

25 "Bombay, like a practitioner of yoga, was folding in on itself, as its centre moved from the south, where there was no room to grow, to this swamp land near the airport. New financial buildings were opening every month [...] American Express, ICICI Bank, HSBC, Citibank, you name it - and the lucre in their vaults, like butter on a hot plate, was melting and trickling into the slums, enriching some and scorching others among the slum-dwellers."

26 "[...] things had changed. It was now a season of will power in Bombay: the coalition of corruption, philantropy, and inertia that had protected them for so long was disintegrating. A new official had been put in charge of clearing the city's illegal slums. He had smashed miles of huts in Thane and promised to do the same in Mumbai."

27 “[...] the government is starting redevelopment in Dharavi. Asia's biggest slum will become Asia's richest slum. This area is boiling with money."

"God has visited the slums today." 
di mantenere il controllo sui processi dell'accumulazione materiale e culturale della città. (Varma 2012: 16$)^{29}$

In Last Man in Tower si racconta di una Mumbai diventata capitale globale (Sassen 1991), connessa con le altre realtà finanziarie e tecnologiche del pianeta, a discapito però della vecchia concezione nerhuviana, che aveva creduto nella possibile convivenza in città tra le tante diverse comunità etniche e religiose.

Per costruire i suoi due ultimi grattacieli dal nome esotico, lo Shangai e il Milano, un costruttore deve ottenere il consenso dei residenti di un condominio sopravvissuto ai suoi giorni migliori, la Vishram Society. Questa impresa si presenta però difficile. La Vishram Society è riottosa al cambiamento: la comunità dei suoi abitanti è piuttosto coesa, la rete di relazioni tra famiglie e individui è piuttosto fitta. Tutti provenienti da regioni diverse ma ormai radicati a Bombay, induisti, cristiani e mussulmani non hanno attriti di natura religiosa: a Vishram vige l'ethos secolarizzato della comunità multiconfessionale.

Un eczema di divinità azzurrognole, dèi barbuti e Cristi alonati ricoprivano la porta dell'interno $3 \mathrm{~b}$, un lascito di generazioni di condomini ecumenici che avevano ognuno aggiunto qualche immagine della propria fede senza rimuovere quelle degli altri, così da rendere impossibile capire se l'occupante di turno fosse induista, cristiano o membro di un qualche culto sincretistico praticato solo in quel palazzo. (Adiga 2012: 15) ${ }^{30}$

Ma le esigenze della nuova economia liberista, con il suo corollario di darwinismo sociale, facendo leva sulle paure di sempre finisce per costruire una nuova sensazione diffusa di insicurezza. Gradualmente le convinzioni e gli scrupoli dei condomini si allentano e alla solidarietà si sostituisce inesorabilmente la diffidenza, soprattutto verso gli ultimi arrivati.

'Troppe persone arrivano in questa città! e tutti vogliono succhiare dalle nostre...' diceva la signora Puri toccandosi il petto. La Battaglia le rispondeva: 'E voi, signora, siete caduta qui a Bombay dal cielo? La vostra famiglia non era di Delhi?' 'I miei genitori erano di Delhi, io sono nata qui e poi allora c'era più spazio. Ora è tutto pieno. [...] gli stranieri devono smetterla di continuare ad arrivare... (39) ${ }^{31}$

29 "[...] in which slums and shanty-towns are cleared for office blocks and shopping mall, where agricultural land is appropriated for ever-expanding urban conurbations and where a new managerial and technocratic elite overlaps with older elites seeking to maintain control of the processes of material and cultural accumulation in the city."

30 "An eczema of blue-skinned gods, bearded godmen, and haloed Christs covered the metal door of 3B - a testament to generations of ecumenical tenants who had each added a few icons of their own faith without removing those of any other - so that it was impossible to know if the present tenant was Hindu, Christian, or a member of a Hybrid cult practised only in this building."

31 “'Too many people come into the city, it's a fact,' Mrs Puri said. 'Everyone wants to suck on our ...' She touched her breasts. The Battleship turned to her.'And did you drop to Bombay from heaven, Msr Puri? Isn't your family from Delhi?'My parents were born in Delhi, Mrs Rego, but I was born right here. There was enough space in those days. Now it's full. [...] outsiders should stop coming here." 
Manco a dirlo, il costruttore riuscirà nel suo intento disgregando la comunità condominiale che, pur di accettare le offerte in denaro, non esiterà a trasformarsi da comunità solidale in una cricca di assassini per fare fuori l'unico residente che si oppone al piano di ristrutturazione. Un professore di storia, che offre il doposcuola gratis ai figli dei suoi condomini, dapprima amato e rispettato da tutti ma col tempo considerato, per il suo ostinato rifiuto, l'ostacolo a tutti i sogni di realizzazione degli abitanti dell'intero fabbricato. Chi vuole una badante per il figlio handicappato, chi vuole raggiungere i figli in America, chi vuole traslocare in una zona migliore, chi vuole riscattarsi da una vita da perdente, chi semplicemente non regge al clima di generalizzata ostilità che l'opportunità di arricchimento ha ingenerato. Così l'ultimo uomo sulla torre, verrà buttato giù proprio dalla torre dai suoi cari vicini di casa.

Last Man in Tower è la storia di un assassinio, effettuato non dagli scagnozzi del costruttore che, sicuro di sé, rifiuta di sporcarsi le mani ("quei giorni sono ormai andati, i banditeschi giorni degli anni '80 e '90”, 289) 32, ma dai suoi rispettabili coinquilini, a testimonianza che la corruzione non è più di esclusiva pertinenza malavitosa o affaristica ma è penetrata nei cuori della gente comune e cosiddetta per bene. Malmenato e imbavagliato, il professore verrà buttato giù dalla sommità del vecchio malandato, amato condominio. L'accurata inchiesta sulla sua morte si concluderà con un equanime verdetto di suicidio da sindrome depressiva. Un ostacolo rimosso alla progressiva opera di "riqualificazione" della città. Last Man in Tower racconta con amara e feroce ironia la crisi profonda di un'idea di città, la città del melting pot indiano, che finisce per cedere il passo alle ragioni di una new economy globalizzata che sempre più invasivamente può travolgere e strappare il tessuto civile e sociale di una qualsiasi comunità condominiale così come dell'intera città alle prese con le infinite difficoltà della convivenza di decine di milioni di persone. La battaglia è in corso, la guerra è appena cominciata.

\section{Riferimenti bibliografici}

Adiga, A. (2011). Last Man in Tower. London: Atlantic Books.

Appadurai, A. (2000). Spectral Housing and Urban Cleansing: Notes on Millennial Mumbai. Public Culture, 12 (3), 627-651.

Boo, K. (2012). Behind the Beautiful Forevers: Life, Death, and Hope in a Mumbai Undercity. New York: Random House.

Brickell, K., \& Datta, A. (Eds.). (2011). Translocal Geographies: Spaces, Places, Connections. Burlington: Ashgate.

Chandra, V. (2007). Sacred Games. London: Faber \& Faber.

Ciocca, R. (2008). Mother India and Paradise Lost: Myth, History, and Fiction in the City of Mumbai. Anglistica. An Interdisciplinary Journal, 12 (2), 105-119.

Cloke, P., \& Johnston, R. (Eds). (2005). Spaces of Geographical Thought: Deconstructing Human Geography's Binaries. London: Sage. 
Dawson Varughese, E. (2013). Reading New India. Post-illennial Indian Fiction in English. London: Bloomsbury.

Freitag, U., \& Von Oppen, A. (Eds.). (2010). Translocality: The Study of Globalising Processes from a Southern Perspective. Leiden: Brill.

Gopal, P. (2009). The Indian English Novel, Nation, History and Narration. Oxford: Oxford University Press. Khilnani, S. (2003). The Idea of India. London: Penguin.

Mehta, S. (2005). Maximum City. Bombay Lost and Found. London: Review.

Mishra, V. (2002). Bollywood Cinema. Temples of Desire. London and New York: Routledge.

Nagarkar, K. (1996). Ravan and Eddie. New Delhi: Penguin India.

Prakash, G. (2010). Mumbai Fables. A History of an Enchanted City. Princeton and Oxford: Princeton University Press.

Pratt, M. L. (1992). Imperial Eyes. Travel Writing and Transculturation. London: Routledge.

Roberts, G. D. (2004). Shantaram. London: Abacus.

Rushdie, S. (1996). The Moor's Last Sigh. London: Vintage.

Sassen, S. (1991). The Global City, Princeton: Princeton University Press.

. (2005). The Global City: Introducing a Concept. Brown Journal of World Affairs, 11 (2), 27-43.

Varma, R. (2012). The Postcolonial City and its Subjects, London, Nairobi, Bombay. London and New York: Routledge. 